\title{
Performance Evaluation of Wireless Sensor Networks Based on Hesitant Fuzzy Linguistic Preference Relations
}

\author{
https://doi.org/10.3991/ijoe.v14i05.8650 \\ Wei Zhai \\ Xi'an Aeronautical University, Xi'an, China \\ 2587842805 @qq. com
}

\begin{abstract}
This paper aims to pinpoint the wireless sensor network (WSN) with the fewest sensor faults through performance evaluation. For this purpose, a WSN performance evaluation method was put forward based on hesitant fuzzy linguistic preference relations (HLPRs). Then, the basic operations and comparison rules were introduced in details. The proposed method was tested in a real case. The results show that the method enables the decision maker to express his exact opinions on different WSN alternatives, and performs well in evaluating WSNs with uncertain information. The research findings shed new light on the selection, performance selection, and promotion of WSNs.
\end{abstract}

Keywords-wireless sensor network (WSN), hesitant fuzzy linguistic preference relations (HLPRs), performance evaluation, sensor faults.

\section{Introduction}

Open the document you would like to format and import the styles. How this works depends very much on the version of MS WORD that you use. The styles' names to be used for online-journals.org are preceded by a "0." which makes them appear first in the styles list and therefore easier to be found. As a symbol of the science development, the wireless sensor network (WSN) is a multi-hop selforganization network constructed by a specific distributed protocol to detect, collect, process and transfer information via low-cost, energy-efficient small sensor nodes [1]. It is independent of the base station, mobile router and other basic telecom infrastructure. Through the collaboration with other WSNs, a WSN supports real-time, allweather and ubiquitous detection, monitoring and collection of information, especially a huge amount of reliable information [2]. Of course, the WSN also has its downside. Fault is one of the major problems with the WSN [3]. In reality, the fault varies from one network to another. Hence, it is very meaningful to find a proper alternative to the WSN to minimize the effect of fault [4-5]. In light of the fault features, a number of alternatives have been constructed to contain the fault of the WSN [6-7]. Thus, the next step is to select the optimal alternative out of the existing ones.

Facing the selection problem, this paper presents an evaluation method for WSN performance based on hesitant fuzzy linguistic preference relations (HLPRs), and lays down the basic operations and comparison rules. The method helps the decision mak- 
er provide his/her opinions on different WSN alternatives. Then, the proposed method was applied in a real case to verify its effect.

The remainder of this paper is organized as follows: Section 2 introduces the relevant concepts of WSN fault analysis; Section 3 describes the pairwise method called HLPRs; Section 4 verifies the effect of the proposed method through a real case; Section 5 wraps up this paper with some meaningful conclusions.

\section{Relevant Concepts of WSN Fault Analysis}

As mentioned above, the WSN is a multi-hop self-organization network of some low-cost small sensor nodes in the detected region. The wireless sensor nodes can collect a large number of data and information, and send them back to the network via wireless connections [3]. The WSN overcomes the drawbacks of the traditional way of tracking detection [8]. In general, the WSN consists of wireless sensor nodes, perception targets and observers, and wireless communication paths linking up these entities. The main components of the network include power supply, sensor module, processing module, communication module, software, special positioning sensors, shifting module and self-contained electric system. The WSN integrates communication technology and communication technology into an organic whole, marking a major change in the information era. Figures $1 \sim 2$ illustrates a WSN consisting of sensor nodes, sink nodes and users.

The wireless sensor nodes in the sensor module mainly perceive and collect the environmental information in a specific field, namely, temperature, humidity and vibration. The sink nodes, as a special type of sensor nodes, sort out and unload the perceived information, and then transmit it to the Internet or other external networks. With no power limit, the sink nodes boast stronger computing, storage and communication capacities than the common nodes.

The WSN is known for its low cost, energy efficiency, high operability and selforganization ability. Recent years has seen the proliferation of the network from the military domain (e.g. battleground positioning and WMD monitoring) to agriculture (crop monitoring), disaster prevention (forest fire and flood monitoring), and healthcare (senior care, home healthcare, and chronic disease monitoring). In addition, the design and implementation of routing protocol has been a hot topic in WSN research. The previous studies have shown that a multi-hop network can accept more faults than a single-hop network.

Of course, the WSN also has its limitations. As a distributed computing platform, the WSN inevitably suffers from the lack of storage space, electric power, CPU capacity and other resources. What is worse, the wireless sensors, often left unattended in extreme environments, may fail under external disturbances. The problem should not be overlooked before applying wireless sensors in more fields.

The WSN faults can be divided into different categories, depending on the classification criterion. In terms of time, the faults are categorized as permanent fault, temporary fault and transient fault; in terms of causes, the faults are split into software fault, hardware fault, and other faults. Each type of fault has its unique impacts, and should 
be treated with a specific measure. For example, a permanent fault must be solved manually, while a temporary fault can be handled automatically. Therefore, the WSN alternatives should be selected based on the features of different faults.

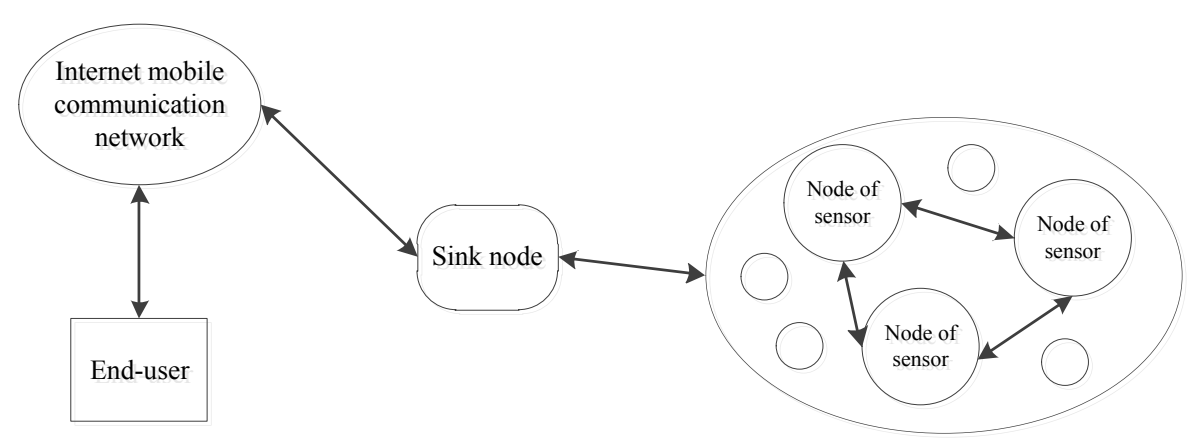

Fig. 1. The simple introduction of wireless sensor network

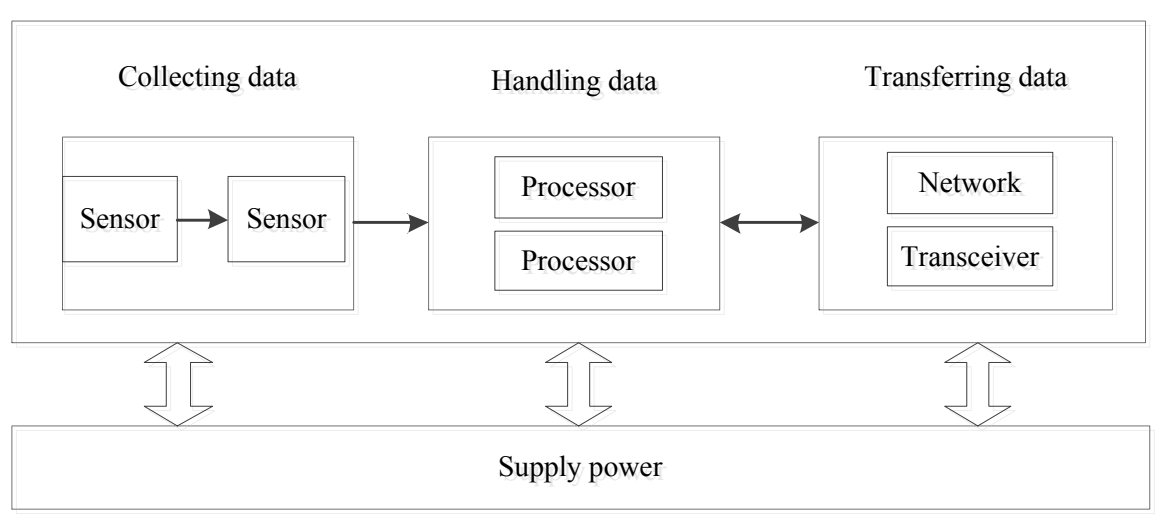

Fig. 2. The structure of the nodes of wireless sensor network

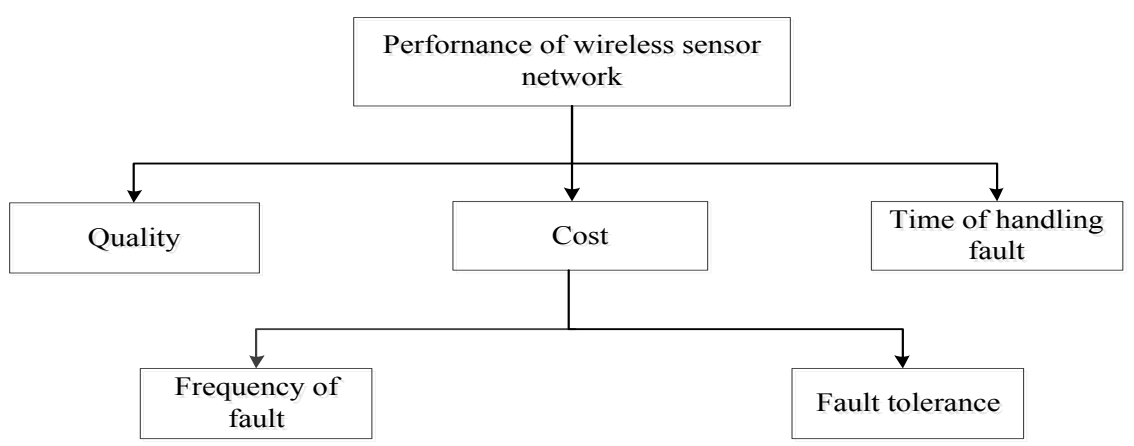

Fig. 3. The attributes of assessing reliability of wireless sensor network 


\section{WSN Selection Method}

The WSN evaluation system usually contains much more qualitative information than quantitative information, adding to the difficulty in information expression by the decision maker. To solve the problem, some information expression methods have been developed, such as 2-tuple linguistic fuzzy set, linguistic fuzzy preference relations, intuitionistic fuzzy linguistic term set, and the HLPRs. Among them, the HLPRs is a typical pairwise comparison method. Relying on the method, the decision maker can express his/her preferences towards difference WSN alternatives from different aspects or attributes [9-10]. Below is a detailed description of the basic concepts, the main algorithm and the alternative rating procedure of the HLPRs.

The hesitant fuzzy linguistic term (HFT) set was proposed by Rodriguez et al. in 2012. The original definition goes as follows.

Definition 1. [9] Let $L T=\left\{t_{0}, \ldots, t_{T}\right\}$ be a linguistic term set. Then, an HFT set $H_{F T}$ can be created based on an ordered set of the consecutive general linguistic terms. However, the HFT set in this definition does not reflect the common thinking pattern, and thus may not be suitable for our research.

Hence, a new definition was proposed in the mathematical form [11-12], which is more applicable to the evaluation problem.

Definition 2. [11] Let $Z=\left\{z_{1}, z_{2}, \ldots, z_{N}\right\}$ be a fixed set, and $H S=\left\{h t_{-\tau}, \ldots, h t_{-1}, h t_{0}\right.$, $\left.h t_{1}, h t_{2}, \ldots, h t_{\tau}\right\}$ be a linguistic term set. Then, a common HFT in $Z$ can be defined as:

$$
H T=\left\{\left\langle z_{i}, h t\left(z_{i}\right)\right\rangle \mid z_{i} \in Z\right\}
$$

where $h t\left(z_{i}\right)$ is the possible membership degrees of number $z_{i} \in Z$ to the set $Z$ and can be rewritten as $h t\left(z_{i}\right)=\left\{h t_{\delta i}\left(z_{i}\right)\right\}$, with $\delta_{i}$ being the subscript of a specific membership degree of the HFT.

Suppose the decision maker provides a set of 9 linguistic terms to describe the real problem below. Note that the set is exhaustive and exclusive. The linguistic terms are symmetrically distributed on the left and the right to the central linguistic term.

$$
H T=\left\{\begin{array}{l}
h t_{-4}=\text { verymuch bad, } h t_{-3}=\text { very bad, } \\
h t_{-2}=\text { slightly bad }, \quad h t_{-1}=\text { bad }, \\
h t_{0}=\text { medium, } h t_{1}=\text { good, } \\
h t_{2}=\text { slightly good, } h t_{3}=\text { very good, } \\
h t_{4}=\text { verymuch good }
\end{array}\right\} .
$$

Based on this set, the decision maker can express his/her preferences as:

$H T_{1}=\left\{h t_{-4}=\right.$ verymuch bad, $h t_{-3}=$ verybad, $h t_{-2}=$ slightly bad $\}$

The expression can be rewritten as: $\left\{h t_{-4}, h t_{-3}, h t_{-2}\right\}$.

In general, preference relations are either additive preference relations or multiplicative preference relations. Suppose there is a set of number $Z=\left\{z_{1}, z_{2}, \ldots, z_{N}\right\}$ and a preference relation matrix $\mathrm{RR}=\left(r r_{i j}\right)_{n \times n}$ with $r r_{i j} \in[0,1]$. The former can be expressed as: 
$r r_{i j}+r r_{j i}=h t_{0}$

The latter can be expressed as:

$r r_{i j} r r_{j k} r r_{k i}=r r_{j i} r r_{i k} r r_{k j}$

According to the above preference relations, especially additive consistent preference relations, the HFT preference relations can be obtained as follows.

Definition 3. Let RR $=\left(r r_{i j}\right)_{n \times n}$ be a set of HFT preference relations and $H S=\left\{h t_{-\tau}\right.$, $\left.\ldots, h t_{-1}, h t_{0}, h t_{1}, h t_{2}, \ldots, h t_{\tau}\right\}$ be a linguistic term set. According to Equation (2), we have:

(1) If $r r_{i j}=h t_{0}$, there is no difference between alternative $r r_{i}$ and alternative $r r_{j}$; (2) If $r r_{i j}>h t_{0}$, alternative $r r_{i}$ is better than alternative $r r_{j}$; (3) If $r r_{i j}<h t_{0}$, alternative $r r_{j}$ is better than alternative $r r_{i}$.

By Definition 3, the matrix can be constructed below using HFT preference relations:

$$
\begin{array}{rllll} 
& r r_{1} & r r_{2} & r r_{3} & r r_{4} \\
r r_{1} & h t_{11} & h t_{12} & h t_{13} & h t_{14} \\
H F T=r r_{2} & h t_{21} & h t_{22} & h t_{23} & h t_{24} \\
r r_{3} & h t_{31} & h t_{32} & h t_{33} & h t_{34} \\
r r_{4} & h t_{41} & h t_{42} & h t_{43} & h t_{44}
\end{array}
$$

where $h t_{i j}$ is $r r_{i j}$ with HFT preference relations. Since the consistent HFT preference relations can be achieved in multiple ways, the most robust method is to find the average of all possible ways. The possible methods are depicted in Figures 4. In fact, the method can be generated by the decision maker or based on the historical information related to the problem.
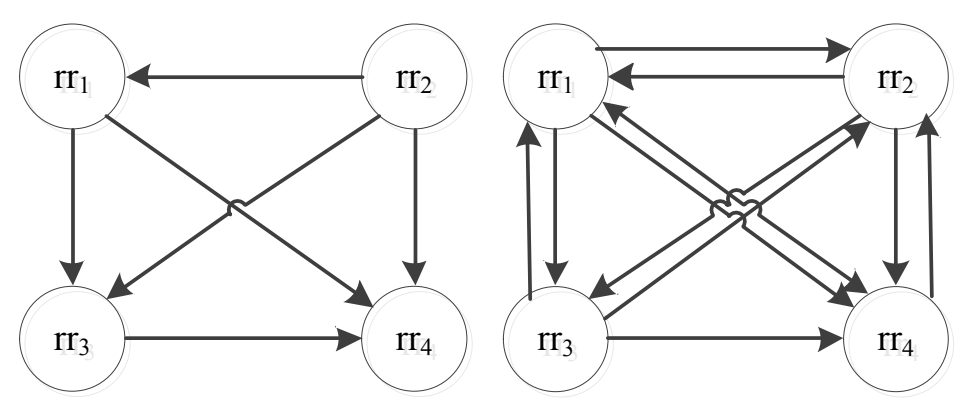

Fig. 4. The explanation of the original consistency (left) and the proposed consistency (right)

Since HFT preference relations include several possible membership degrees, it is important to ensure that the number of each HFT preference relation is the same after aggregating all HFT preference relations. To this end, some principles or rules have been presented by scholars. 
(1) If the decision maker prefers the bigger membership degree, then the smaller number of membership degrees will be extended by adding the largest value of all membership degrees; (2) If the decision maker prefers the smaller membership degree, then the smaller number of membership degrees will be extended by adding the smallest value of all membership degrees; (3) If the decision maker does not provide exact preference information, then the smaller number of membership degrees will be extended by adding the average value of all membership degrees.

After generating the consistent matrix of HFT preference relations, the next step is to aggregate these preference relations from several perspectives (attributes or criteria). This calls for the creation of a proper aggregation method. In reference to previous research, the operational rule of general linguistic term set is introduced first:

(1) $h t_{a} \oplus h t_{b}=h t_{b} \oplus h t_{a}=h t_{a+b}$; (2) $h t_{a} \otimes h t_{b}=h t_{b} \otimes h t_{a}=h t_{a b}$; (3) $\lambda h t_{a}=h t_{\lambda a}$; (4) $\left(h t_{a}\right)^{\lambda}=h t_{a \lambda}$; (5) $\left(\lambda_{1}+\lambda_{2}\right) h t_{a}=\lambda_{1} h t_{a} \oplus \lambda_{2} h t_{a} ;(6) \lambda\left(h t_{a} \oplus h t_{b}\right)=\lambda h t_{a} \oplus \lambda h t_{b}$

Following this operational rule, the HFT preference relations can be combined with attribute weights. Taking a basic operator as an example:

$$
O H F=\sum_{i=1}^{n} \oplus w_{i} h t_{\delta i}
$$

The operator can be further defined as follows. First, the operation of two HFT preference relations is developed with the same length.

Let $h t_{1}=\left\{h t_{\delta 1} \mid \delta=1, \ldots, \# h t_{1}\right\}$ and $h t_{2}=\left\{h t_{\delta 2} \mid \delta=1, \ldots, \# h t_{2}\right\}$ be two HFT preference relations, with \# being the length of HFT preference relation. Then, Equation (5) can be expanded into:

$$
h t_{1} \oplus h t_{2}=\mathrm{U}_{h t_{\delta(\mu) 1} \in h t_{1}, h t_{\delta(v) 2} \in h t_{2}}\left\{h t_{\delta(\mu) 1} \oplus h t_{\delta(v) 2}\right\}
$$

It is still difficulty to compare two HFT preference relations. In general, the average of all membership degrees in a HFT preference relation or the distribution value of a HFT preference relation can be defined or calculated to denote an overall value of a HFT preference relation.

\section{$4 \quad$ Application of the HLPRs}

This section evaluates the WSN performance with the HLPRs, aiming to obtain a ranking order and disclose the differences between WSNs.

Firstly, a decision maker was invited to preside over the selection process. Based on Figure 1, the five attributes are denoted as R1, R2, R3, R4 and R5, respectively. The relative weights the decision maker gave to these attributes are $(0.1,0.2,0.25$, $0.15,0.3)$. In light of historical and relevant data, the decision maker provided the

following linguistic terms:

$$
H T=\left\{\begin{array}{c}
h t_{-3}=\text { very low, } h t_{-2}=\text { slightly low, } \\
h t_{-1}=\text { low, } h t_{0}=\text { medium, ht } h t_{1} \text { good }, \\
h t_{2}=\text { slightly good, } h t_{3}=\text { very good, }
\end{array}\right\} ; \text { Sec- }
$$


ondly, the decision maker compared different pairs of the four WSNs, denoted as $\mathrm{W}_{1}$, $\mathrm{W}_{2}, \mathrm{~W}_{3}$ and $\mathrm{W}_{4}$, respectively. The decision matrices are listed in Tables $1 \sim 5$. The consistent preference relations were calculated by Equation (2), seeking to guarantee the rationality of the preference assessments. After that, the preference relations were aggregated by the operator in Equation (5) to generate the overall assessment of each WSN. Finally, the WSNs were ranked as $W_{2}>W_{1}>W_{4}>W_{3}$ by the comparison rule, indicating that the optimal WSN is $W_{2}$.

Table 1. The pairwise preference relationsby the decision maker on the first attribute

\begin{tabular}{|c|c|c|c|c|}
\hline & $W_{1}$ & $W_{2}$ & $W_{3}$ & $W_{4}$ \\
\hline$W_{1}$ & $\left(h t_{0}\right)$ & $\left(h t_{-}, h t_{-1}\right)$ & $\left(h t_{2}, h t_{3}\right)$ & $\left(h t_{3}\right)$ \\
\hline$W_{2}$ & & $\left(h t_{0}\right)$ & $\left(h t_{0}, h t_{1}\right)$ & $\left(h t_{1}, h t_{2}\right)$ \\
\hline$W_{3}$ & & & $\left(h t_{0}\right)$ & $\left(h t_{-3}, h t_{-1}\right)$ \\
\hline$W_{4}$ & & & & $\left(h t_{0}\right)$ \\
\hline
\end{tabular}

Table 2. The pairwise preference relations by the decision maker on the second attribute

\begin{tabular}{|c|c|c|c|c|}
\hline & $W_{1}$ & $W_{2}$ & $W_{3}$ & $W_{4}$ \\
\hline$W_{1}$ & $\left(h t_{0}\right)$ & $\left(h t_{1}, h t_{2}\right)$ & $\left(h t_{-}\right)$ & $\left(h t_{2}, h t_{3}\right)$ \\
\hline$W_{2}$ & & $\left(h t_{0}\right)$ & $\left(h t_{1}, h t_{2}\right)$ & $\left(h t_{0}, h t_{1}\right)$ \\
\hline$W_{3}$ & & & $\left(h t_{0}\right)$ & $\left(h t_{-2}, h t_{-1}\right)$ \\
\hline$W_{4}$ & & & & $\left(h t_{0}\right)$ \\
\hline
\end{tabular}

Table 3. The pairwise preference relations by the decision maker on the third attribute

\begin{tabular}{|c|c|c|c|c|}
\hline & $W_{1}$ & $W_{2}$ & $W_{3}$ & $W_{4}$ \\
\hline$W_{1}$ & $\left(h t_{0}\right)$ & $\left(h t_{-3}, h t_{-2}, h t_{-1}\right)$ & $\left(h t_{2}, h t_{3}\right)$ & $\left(h t_{-2}, h t_{-1}\right)$ \\
\hline$W_{2}$ & & $\left(h t_{0}\right)$ & $\left(h t_{1}, h t_{2}\right)$ & $\left(h t_{-1}\right)$ \\
\hline$W_{3}$ & & & $\left(h t_{0}\right)$ & $\left(h t_{2}\right)$ \\
\hline$W_{4}$ & & & & $\left(h t_{0}\right)$ \\
\hline
\end{tabular}

Table 4. The pairwise preference relations by the decision maker on the fourth attribute

\begin{tabular}{|c|c|c|c|c|}
\hline & $W_{1}$ & $W_{2}$ & $W_{3}$ & $W_{4}$ \\
\hline$W_{1}$ & $\left(h t_{0}\right)$ & $\left(h t_{2}\right)$ & $\left(h t_{-2}, h t_{-1}, h t_{0}\right)$ & $\left(h t_{2}, h t_{3}\right)$ \\
\hline$W_{2}$ & & $\left(h t_{0}\right)$ & $\left(h t_{0}, h t_{1}\right)$ & $\left(h t_{1}, h t_{2}\right)$ \\
\hline$W_{3}$ & & & $\left(h t_{0}\right)$ & $\left(h t_{-2}, h t_{-1}\right)$ \\
\hline$W_{4}$ & & & & $\left(h t_{0}\right)$ \\
\hline
\end{tabular}

Table 5. The pairwise preference relations by the decision maker on the fifth attribute

\begin{tabular}{|c|c|c|c|c|}
\hline & $W_{1}$ & $W_{2}$ & $W_{3}$ & $W_{4}$ \\
\hline$W_{1}$ & $\left(h t_{0}\right)$ & $\left(h t_{0}, h t_{1}\right)$ & $\left(h t_{-1}\right)$ & $\left(h t_{-2}, h t_{-1}\right)$ \\
\hline$W_{2}$ & & $\left(h t_{0}\right)$ & $\left(h t_{-1}\right)$ & $\left(h t_{0}\right)$ \\
\hline$W_{3}$ & & & $\left(h t_{0}\right)$ & $\left(h t_{-2}\right)$ \\
\hline$W_{4}$ & & & & $\left(h t_{0}\right)$ \\
\hline
\end{tabular}




\section{Conclusions}

To minimize the effect of sensor fault on WSN performance, this paper creates a WSN performance evaluation method based on the HLPRs, and lays down the basic operations and comparison rules. The method helps the decision maker provide his/her opinions on different WSN alternatives, and supports the WSN performance evaluation with uncertain information. Through a case study, it is clear that the proposed method has achieved the desired effect.

\section{References}

[1] Teng, L.P. (2012). Overview of security in wireless sensor networks. Computer Security, 11: 24-29.

[2] Ma, Z.C., Sun, Y.N., Mei, T. (2004). Survey on wireless sensor networks. Journal of China Institute of Communications, 25(4): 114-124.

[3] Yick, J, Mukherjee, B., Ghosal, D. (2008). Wireless sensor network survey. Computer Networks, 52(12): 2292-2330. https://doi.org/10.1016/j.comnet.2008.04.002

[4] Zhou, H. (2014). A Bayes networks inference based method on fault link location in WSNs. Beijing University of Posts and Telecommunications.

[5] Liu, G.L. (2014). The research on fault detection in wireless sensor networks based on data feedback. Xidian University.

[6] Chen, L.S., Guo, Z.S. (2015). Research on ascs system fault diagnosis of automobile automated mechanical transmission, Academic Journal of Manufacturing Engineering, 14(4), 25-33.

[7] Zhou, B. (2009). A study on fault diagnosis in wireless sensor networks. Hangzhou Dianzi University.

[8] Huang X. (2014). Research of wireless sensor network performance testing and intelligent fault diagnosis technology. Shandong University.

[9] Dong, Y.C. (2008). Multi-person decision making under linguistic environment. Xi'an Jiaotong University, 2008.

[10] Wang, H., Xu, Z.S. (2015). Some consistency measures of extended hesitant fuzzy linguistic preference relations. Information Sciences, 297(10): 316-331. https://doi.org/10.1016/j.i ns.2014.10.047

[11] Rodriguez, R.M., Martinez, L., Herrera, F. (2011). Hesitant fuzzy linguistic term sets for decision making. IEEE Transaction on Fuzzy Systems, 29: 109-119.

[12] Zhu, B., Xu, Z.S. (2013). Consistency measures for hesitant fuzzy linguistic preference relations. IEEE Transaction on Fuzzy Systems, 5: 35-45.

\section{$7 \quad$ Author}

Wei Zhai received the B.E. degree in electronic information engineering from Xi' an University of Technology, Xi'an, China, in 2003, the M.E. degree in communications engineering from Xi'an Jiaotong University, Xi'an, China, in 2012. Since 2003, she has been on the faculty of Xi'an Aeronautical University. She is manly engaged in the research of electrons and communication.

Article submitted 30 March 2018. Final acceptance 04 May 2018. Final version published as submitted by the author. 